\title{
林窗中植物竞争强度随林窗发育的变化
}

\author{
向言词 ${ }^{12} \quad$ 彭少麟 $^{1} \quad$ 蔡锡安 $^{1} \quad$ 任 $\quad$ 海 $^{1} \quad$ 周厚诚 $^{3}$ \\ （1 中国科学院华南植物研究所, 广州 510650) （2 湖南科技大学生命科学系, 湘潭 411201)
}

(3 广东省海洋资源发展研究中心, 广州 510070)

摘 要 通过野外移植外来种尾叶桉 (Eucalyptus urophylla)、大叶相思 (Acacia auriculaeformis) 和本地种荷木 (Schima superba) 3 种幼苗 挖沟排除根竞争和砍树创造林窗排除地上竞争的实验, 来测定林窗形成后植物地下竞争和地上 竞争强度的变化以及这些竞争对 3 种幼苗生长的影响。在林窗内, 尾叶桉、大叶相思和荷木受到的地下竞争强度 和地上竞争强度均呈现出随林窗年龄的增长而逐渐增大的趋势。外来种尾叶桉和大叶相思受到的地上竞争强度 大于本地种荷木受到的地上竞争强度, 而荷木受到的地下竞争强度大于两种外来种受到的地下竞争强度。在只有 地下竞争和地上竞争时, 这 3 种幼苗的相对生长速率均小于没有竞争时, 这反映了地下竞争和地上竞争对 3 种幼苗 的生长有抑制性影响，而且这种影响在本地种和外来种间存在差异。

关键词 林窗 地上竞争 地下竞争 相对生长速率

\section{CHANGES IN PLANT COMPETITION WITH THE DEVELOPMENT OF GAPS}

\author{
XIANG Yan-Ci ${ }^{1}{ }^{2}$ PENG Shao-Lin ${ }^{1}$ Cai Xi- $\mathrm{An}^{1}$ REN Hai ${ }^{1}$ and ZHOU Hou-Cheng ${ }^{3}$ \\ (1 South China Institute of Botany , the Chinese Academy of Sciences , Guangzhou 510650 ,China) \\ (2 Department of Biology Hunan University of Science and Technology, Xiangtan 411201 , China) \\ (3 Guangdong Center for Marine Resource R \& D , Guangzhou 510070 ,China)
}

\begin{abstract}
Juvenile plants in a community usually compete with the established vegetation within that community. In general, plants are likely to compete for three kinds of essential resources : photosynthetically active radiation, water and essential nutrients. After the formation of treefall gaps, the competition between plants in gaps and in adjacent understory may change accordingly. Two fundamental questions are how the intensity of competition on juvenile plants changes with the development of gaps and whether the intensity of competition on different species is different or not. We used a field experiment to measure the intensity of competition on juvenile trees in a mixed forest community with infertile soil on an island in Southeastern China. We separated competition into its above- and belowground components in the field. We reduced aboveground competition by felling large trees to create three treefall gaps, and removed belowground competition by trenching. Thus we created three neighborhood treatments : with roots of neighbors only, with shoots of neighbors only and with no neighbors. Seedlings of three tree species were transplanted into the three gaps with and without trenches cut around the gaps. These species included the two exotic species Eucalyptus urophylla S.T. Blake and Acacia auriculaeformis A. Cunn., and one native species, Schima superba Gardn. et Champ. We measured the biomass, the relative growth rate, and competition intensity of seedlings of the three species over two years.

Results based on the average of the three species showed that the intensity of above- and belowground competition on the seedlings of the three species increased gradually with the development of gaps. The intensity of aboveground competition was greater for the two exotic species than that for the native. However, for the native species, the intensity of belowground competition was greater than that for the two exotics. The relative growth rates of seedlings of the three species with roots of neighbors only and with shoots of neighbors only were all less than with no neighbors. The results showed that the competition of shoots and roots of neighbors in gaps imposed negative impacts on the growth of the three species. The impacts differed between exotic and native species.
\end{abstract}

Key words Gap , Aboveground competition , Belowground competition , Relative growth rate

林窗形成后, 林窗内的环境条件发生了变化，

不同的树种会产生不同的反应; 林窗周围的树木侧 
枝不断向林窗内生长 林窗的透光率随之而变 ;那么 林窗中的幼苗生长同样产生变化, 这样林窗内的各 种竞争强度也可能发生变化。

在植物幼苗生长的过程中, 群落中已定居的植 物会与这些幼苗不断竞争。目前, 主要是通过挖沟 和林窗来研究地下根的竞争和地上枝叶的竞争对植 物幼苗生长的影响, 这方面已有许多研究报道 (Strutt \& Keddy , 1996 ; Coomes \& Grubb , 1998 ;2000 ; Lewis \& Tanner , 2000) ,而国内有关竞争对幼苗生长 影响的研究报道较少见。有关南亚热带海岛森林群 落林窗内的竞争及这些竞争对幼苗生长影响的研究 没有相关报道。本文就竞争对外来种大叶相思 ( $A$ -

cacia auriculaeformis A. Cunn.)、尾叶桉 (Eucalyptus urophylla S. T. Blake) 和本地种荷木 ( Schima superba Gardn. et Champ） 3 种幼苗的影响作对比研究, 以此 了解植物外来种入侵的限制因子。

\section{1 样地概况与研究方法}

\section{1 研究样地}

南澳岛地处北纬 $23^{\circ} 23^{\prime} 33^{\prime \prime} \sim 23^{\circ} 29^{\prime} 11^{\prime \prime}$, 东经 $116^{\circ} 56^{\prime} 24^{\prime \prime} \sim 117^{\circ} 08^{\prime} 59^{\prime \prime}$ 。岛上的原生性植被为南亚 热带常绿阔叶林。由于长期的人为活动干扰, 其原 生性植被多已不存在, 仅在局部地段如村边或庙旁 作为 风水林” 保存下来的小面积次生林。现有植被 类型中分布面积较大的为马尾松林、尾叶桉林、大叶 相思林及其混交林, 这些都是人工林。

研究样地位于南澳岛黄花山林场附近的人工混 交林群落内 海拔 $241 \mathrm{~m}$, 西北坡向 坡度 $3^{\circ}$, 群落高 度约 $7 \mathrm{~m}$, 群落可分为乔木层、灌木层和草本层, 结 构较复杂, 郁闭度较大, 土壤为花岗岩发育成的红壤 土, 群落中主要植物有大叶相思、梅叶冬青 (Ilex asprella $)$ 和短叶㯟( Panicum brevifolium)。

\section{2 研究方法}

1) 1999 年 1 月 26 日, 在群落中选择达到林冠 层而冠幅面积相近的乔木, 将其砍倒而创造 3 个近 似椭圆形的林窗 A、B 和 C, 林窗面积按椭圆面积公 式计算。林窗长轴与坡向大致垂直, 3 个林窗面积 各约为 $160 \mathrm{~m}^{2}$ 。为了维持 $\mathrm{A}$ 和 $\mathrm{B}$ 林窗大小不变, 在 1999 2000 这两年中，每个季度都把林窗周围伸入 林窗内的侧枝剪除, C 林窗则不剪除伸入林窗内的 侧枝。为了排除根的竞争, 在 $\mathrm{A}$ 和 $\mathrm{C}$ 林窗内的样地 周围挖宽 $20 \mathrm{~cm}$ 、深 $50 \mathrm{~cm}$ 的沟 (距样地 $20 \mathrm{~cm}$ ) ,每年 7 月和 9 月把冲入沟中的泥土移开, 并剪除从样地 外伸入沟中的根; 为了作对比研究, $\mathrm{B}$ 林窗内的样地
周围不挖沟。在 $\mathrm{A} 、 \mathrm{~B}$ 和 $\mathrm{C}$ 林窗内各选 1 块 $12.4 \mathrm{~m}$ $\times 10 \mathrm{~m}$ 的样地, 分成 $4 \mathrm{~m} \times 10 \mathrm{~m}$ 的 3 块小样地 $\mathrm{P}_{\mathrm{E}}$ 、 $\mathrm{P}_{\mathrm{A}} 、 \mathrm{P}_{\mathrm{S}}$, 在小样地间挖两条宽 $0.2 \mathrm{~m}$, 长 $10 \mathrm{~m}$ 和深 5 $\mathrm{cm}$ 的小沟把这 3 块小样地隔开, 在 1999 年 4 月在小 样地 $\mathrm{P}_{\mathrm{E}} 、 \mathrm{P}_{\mathrm{A}} 、 \mathrm{P}_{\mathrm{S}}$ 分别种上尾叶桉、大叶相思和荷木幼 苗, 苗株间距为 $50 \mathrm{~cm} \times 50 \mathrm{~cm}$, 则 3 种幼苗的总数各 为 160 株。为了有利于幼苗的存活, 在移植幼苗前, 林窗内样地上的灌丛和草本层植物均剪除掉。野外 实验进行时间从 1999 年 1 月 26 日到 2000 年 12 月 底。

2) 生物量的测定:1999 年 4 月把幼苗移栽到样 地中 移栽前每种幼苗均随机抽取并测定 60 株的生 物量 取平均值作为每种幼苗的初始生物量 $M_{1}$ (时 间为 $t_{1}$ )。在 1999 年 7 月 15 日、9 月 22 日、12 月 15 日、 2000 年 4 月 27 日、7 月 15 日、10月 30 日、12 月 25 日各挖出 6 株幼苗测定其生物量 $M_{2}$ (时间为 $\left.t_{2}\right)$ 。

$3)$ 相对生长速率按以下公式计算: $R=[\ln$ $\left.\left(M_{2} / M_{1}\right)\right] / d$, (单位 $: \ln \left(\mathrm{g} \mathrm{g}^{-1}\right) \cdot \mathrm{d}^{-1} ; M_{1} 、 M_{2}$ 分别 是时间为 $t_{1} 、 t_{2}$ 时的生物量, $d$ 是 $t_{2}$ 与 $t_{1}$ 之间的生 长日数)。

4) 净初级生产力的计算 : $N P P=\left(M_{2}-M_{1}\right) / d$ (单位: $\mathrm{g} \mathrm{m}^{-2} \mathrm{~d}^{-1}$ )。

5) 竞争强度的计算 林窗内幼苗所受到的地下 根竞争强度 $(\mathrm{B})$ 和地上枝叶竞争强度 $(A)$ 按以下公 式计算 (Wilson \& Tilman, 1993$): B=\left(N P P_{1}-\right.$ $\left.N P P_{3}\right) / N P P_{1}, A=\left(N P P_{1}-N P P_{2}\right) / N P P_{1}\left(N P P_{1}\right.$ 是 $A$ 林窗内没有地下根和地上枝叶竞争情况下幼苗的 净初级生产力, $N P P_{2}$ 是 $C$ 林窗内只有地上枝叶竞 争时幼苗的净初级生产力, $N P P_{3}$ 是 $B$ 林窗只有根 竞争时幼苗的净初级生产力)。图 2 的相对生长速 率计算时, $M_{2}$ 采用的生物量是 2000 年 12 月 25 日 测定的。用单因素方差法进行差异显著性分析。

\section{2 结果与分析}

\section{1 竞争强度的变化}

林窗形成后，林窗内的地下根和林窗周围的植 物生长会发生变化, 这样一来在林窗内生长的各种 植物幼苗受到地下和地上竞争可能产生相应的变 化。

从图 1 可以看出, 尾叶桉、大叶相思和荷木幼苗 受到的地下根竞争强度均呈现出随林窗年龄的增长 而不断增大。在树倒形成林窗后, 林窗内的根均相 应死亡, 土壤中的根大量减少, 同周围林下相比, 林 

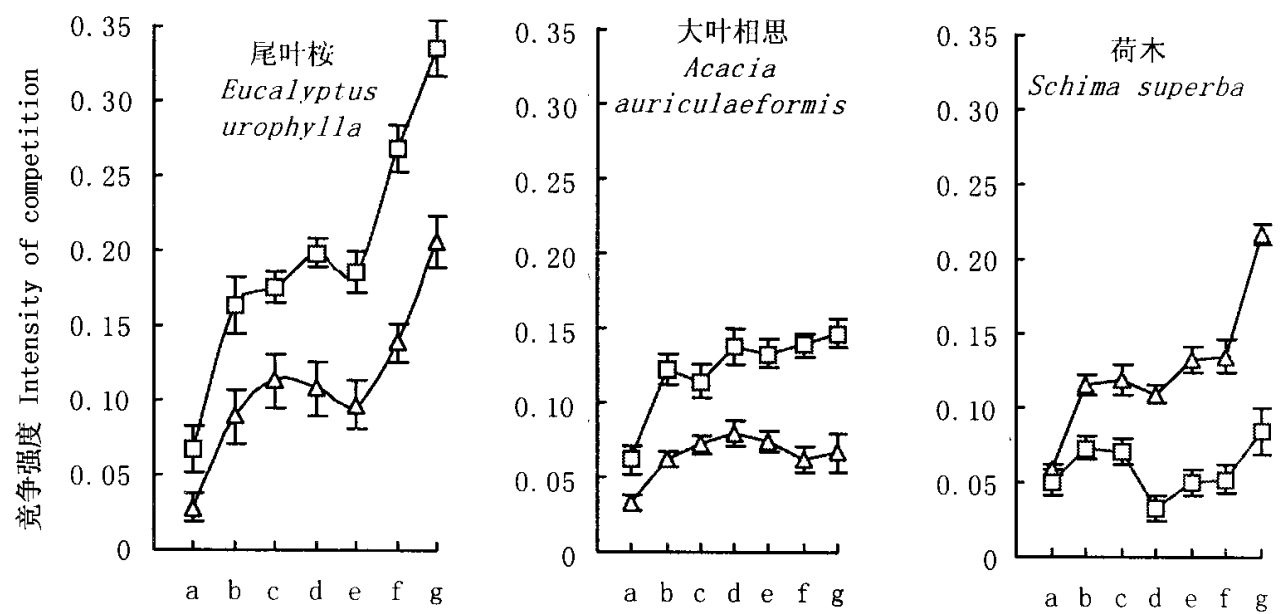

林窗形成时间 Time of gap development（d）

图 1 不同树苗所受到的竞争强度的变化

Fig. 1 Changes in intensity of competition on tree seedlings a : 165 b :238 $\quad$ c : $: 322 \quad$ d : 448 e :534 f $: 640 \quad$ g: $: 696$

$\triangle$ 地下竞争强度 Intensity of belowground competition $\square$ 地上竞争强度 Intensity of aboveground competition

窗内活根极少（Denslow et al，，1998；Ostertag， 1998）, 这样一来, 生长在林窗内的幼苗受到的地下 根的竞争就相应减小; 而随着林窗年龄的增长 林窗 周围植物的根就不断地伸入林窗的土壤中，林窗土 壤中的活根就不断增加，因而生长在林窗内的植物 幼苗受到的地下根的竞争就增强。

这 3 种幼苗在林窗内生长时受到林窗周围的植 物地上枝叶的竞争强度也随着林窗年龄的增长而增 大(图 1)。林窗形成后, 光照增加, 林窗内土壤含水 量高于林窗周围林下土壤, 资源相对丰富 ,( Vitousek \& Denslow , 1986 ; Becker et al . , 1988)，林窗内的竞 争比周围林下要小 林窗周围的树枝侧向生长加快, 伸入林窗内的枝叶不断增多, 这些枝叶同林窗内的 幼苗竞争有效光辐射不断加剧, 这样就造成对林窗 内生长的幼苗地上竞争强度不断增大。

从表 1 可以看出,尾叶桉和荷木两种幼苗受到 的地下竞争强度间的差异不显著 $(p>0.05)$, 而大叶 相思幼苗受到的地下竞争强度分别与这两种幼苗相 比 到后期差异显著 $(p<0.05) ; 165 \mathrm{~d}$ 之后,3 种幼 苗受到的地上竞争强度间的差异都显著 $(p<0.05)$ 。 从竞争强度指数来看 (图 1 , 表 1 ), 尾叶桉受到的地 上竞争强度最强, 其次是大叶相思, 荷木最弱; 而地 下竞争则是荷木受到的竞争最强，其次是尾叶桉，大 叶相思最弱。荷木的耐阴性较强, 而外来种尾叶桉 和大叶相思的耐阴性不如荷木, 所以荷木幼苗受到 的地上竞争要小于两种外来种幼苗; 尾叶桉和大叶 相思适应性强, 在肥力低的贫痛土壤中均能生长较 好,而且大叶相思有生物固氮的能力, 所以两种外来
种尾叶桉和大叶相思幼苗受到的地下竞争强度较 小;而荷木没有这些特性，其受到的地下根的竞争强 度大。

\section{2 相对生长速率}

尾叶桉幼苗的相对生长速率，在没有竞争、只有 地下根竞争和只有地上枝叶竞争时分别为 0.008 459、0.008 175、0.007 682 , 可见尾叶桉幼苗在 有根竞争和有地上枝叶竞争时, 其相对生长速率都 小于没有竞争时，而且这种差异均达到显著水平 $(p$ $<0.05)$;在只有根竞争和只有地上枝叶竞争时，尾 叶桉幼苗的相对生长速率间的差异显著 $(p<0.05)$ (图 2)。

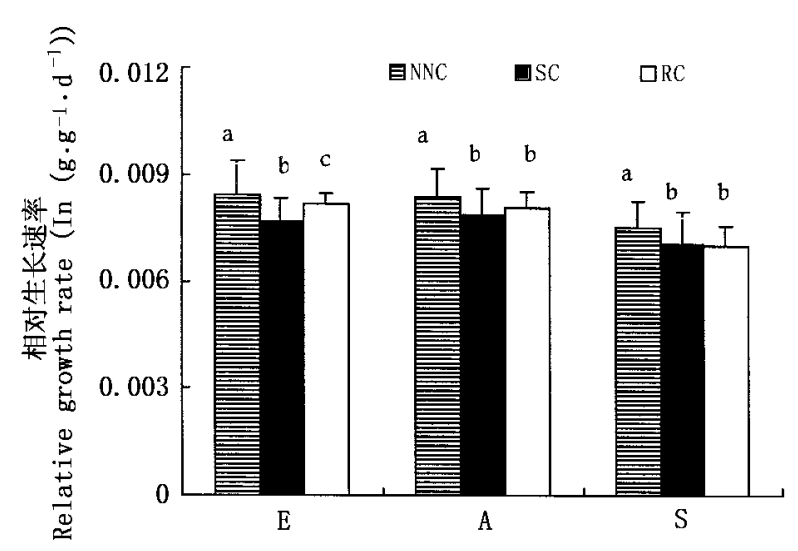

图 2 竞争对幼苗相对生长速率的影响

Fig.2 Impacts of competition on the relative growth rate of seedlings

$\mathrm{E}$ : 尾叶桉 Eucalyptus urophylla A : 大叶相思 Acacia auriculaeformis S : 荷木 Schima superba NNC :无竞争 No neighbor competition $\mathrm{SC}$ : 枝叶竞争 Shoot competition $\mathrm{RC}$ : 根竞争 Root competition

不同字母表示同种的差异显著性 Significance in the same species with different letters $(p<0.05)$ 
表 1 幼苗种间竞争差异 (平均值 \pm 标准差)

Table 1 The difference of interspecific competition (Mean \pm standard deviation)

\begin{tabular}{|c|c|c|c|c|c|c|c|}
\hline \multirow[t]{2}{*}{$\begin{array}{c}\text { 竞争类型 } \\
\text { Type of competition }\end{array}$} & \multirow[t]{2}{*}{$\begin{array}{c}\text { 时间 } \\
\text { Time (d) }\end{array}$} & \multirow[t]{2}{*}{$\begin{array}{c}\text { 尾叶桉 } \\
\text { Eucalyptus } \\
\text { urophylla }\end{array}$} & \multirow{2}{*}{$\begin{array}{c}\text { 大叶相思 } \\
\text { Acacia } \\
\text { auriculaeformis }\end{array}$} & \multirow[t]{2}{*}{$\begin{array}{l}\text { 荷木 } \\
\text { Schima } \\
\text { superba }\end{array}$} & \multicolumn{3}{|c|}{$\begin{array}{c}\text { 种间竞争差异 } \\
\text { Difference of } \\
\text { interspecific competition }\end{array}$} \\
\hline & & & & & E-A & E-S & A-S \\
\hline \multirow{7}{*}{$\begin{array}{l}\text { 地下竞争强度 } \\
\text { Intensity of below- } \\
\text { ground competition }\end{array}$} & 165 & $0.028 \pm 0.009$ & $0.033 \pm 0.005$ & $0.058 \pm 0.003$ & NS & NS & NS \\
\hline & 238 & $0.089 \pm 0.018$ & $0.062 \pm 0.006$ & $0.115 \pm 0.007$ & NS & NS & * \\
\hline & 322 & $0.113 \pm 0.019$ & $0.072 \pm 0.006$ & $0.119 \pm 0.010$ & * & NS & * \\
\hline & 448 & $0.108 \pm 0.018$ & $0.079 \pm 0.008$ & $0.109 \pm 0.006$ & NS & NS & NS \\
\hline & 534 & $0.097 \pm 0.016$ & $0.074 \pm 0.007$ & $0.133 \pm 0.009$ & NS & NS & $*$ \\
\hline & 640 & $0.139 \pm 0.012$ & $0.062 \pm 0.009$ & $0.135 \pm 0.011$ & * & NS & * \\
\hline & 696 & $0.207 \pm 0.018$ & $0.067 \pm 0.013$ & $0.217 \pm 0.007$ & $* * *$ & NS & $* * *$ \\
\hline \multirow{7}{*}{$\begin{array}{l}\text { 地上竞争强度 } \\
\text { Intensity of above- } \\
\text { ground competition }\end{array}$} & 165 & $0.067 \pm 0.016$ & $0.062 \pm 0.009$ & $0.049 \pm 0.008$ & NS & NS & NS \\
\hline & 238 & $0.163 \pm 0.019$ & $0.122 \pm 0.010$ & $0.073 \pm 0.009$ & * & $* *$ & $* *$ \\
\hline & 322 & $0.176 \pm 0.011$ & $0.115 \pm 0.011$ & $0.070 \pm 0.010$ & * & $* *$ & $*$ \\
\hline & 448 & $0.199 \pm 0.009$ & $0.138 \pm 0.012$ & $0.033 \pm 0.008$ & * & $* * *$ & $* *$ \\
\hline & 534 & $0.187 \pm 0.014$ & $0.133 \pm 0.008$ & $0.050 \pm 0.007$ & * & $* * *$ & $* *$ \\
\hline & 640 & $0.269 \pm 0.016$ & $0.138 \pm 0.008$ & $0.053 \pm 0.011$ & $* * *$ & $* * *$ & $* *$ \\
\hline & 696 & $0.336 \pm 0.019$ & $0.147 \pm 0.010$ & $0.085 \pm 0.015$ & $* * *$ & $* * *$ & * \\
\hline
\end{tabular}

$*: p<0.05 \quad * *: 0.01<p<0.01 \quad * * *: p<0.001 \quad$ NS :无显著差异 Non-significance

E-A : Eucalyptus urophylla-Acacia auriculaeformis E-S : Eucalyptus urophylla-Schima superba A-S : Acacia auriculaeformis-Schima superba

大叶相思幼苗的相对生长速率, 在没有竞争、只 有地下根竞争和只有地上枝叶竞争时分别为 0.008 392、0.008 077、0.007858, 后两者与前者的差 异都达到显著水平 $(p<0.05)$,后两者间的差异不显 著 $(p>0.05)$ 。荷木幼苗的相对生长速率, 在没有竞 争、只有地下根竞争和只有地上枝叶竞争时分别为 0.007 549、0.007 $067 、 0.007027$, 后两者与前者差异 显著 $(p<0.05)$, 而后两者间的差异不显著 $(p>$ 0.05 )(图 2)。

在只有地下根竞争时, 尾叶桉与大叶相思两种 幼苗的相对生长速率间的差异不显著 $(p>0.05)$, 而 这两种幼苗分别同荷木相比, 其相对生长速率间的 差异都显著 $(p>0.05)$; 在只有地上枝叶竞争时,3 种幼苗的相对生长速率间两两相比, 其差异显著水 平同只有地下根竞争时相似。这反映了林窗内地下 根竞争和地上枝叶竞争对外来种和本地种幼苗的抑 制性影响有差异(图 2)。

\section{3 结 论}

在林窗内, 尾叶桉、大叶相思和荷木 3 种幼苗受 到地下根竞争强度和地上枝叶竞争强度均呈现出随 林窗年龄的增长而逐渐增大的趋势。在林窗内生长 时, 外来种尾叶桉和大叶相思两种幼苗受到的地上 枝叶的竞争强度大于本地种荷木幼苗, 而荷木幼苗
受到的地下根的竞争强度大于两种外来种幼苗。在 只有地下根竞争和地上枝叶竞争时, 这 3 种幼苗的 相对生长速率均小于没有竞争时, 这反映了地下根 竞争和地上枝叶竞争对 3 种幼苗的生长有抑制性影 响, 而且这种影响在本地种和外来种幼苗间有差异。

\section{参 考 文 献}

Becker, P., P. E. Rabenold, J. R. Idol \& A. P. Smith. 1988. Water potential gradients for gaps and slopes in a Panamanian tropical moist forests dry season. Journal of Tropical Ecology, 4: $173 \sim 184$.

Coomes, D. A. \& P. J. Grubb. 1998. Responses of juvenile trees to above- and belowground competition in nutrient-starved Amazonian rain forest. Ecology, 79: $768 \sim 782$.

Coomes, D. A. \& P. J. Grubb. 2000. Impacts of root competition in forests and woodlands: a theoretical framework and review of experiments. Ecological Monographs, 70: 171 207.

Denslow, J. S., A. M. Ellison \& R. R. Sanford. 1998. Treefall gap size effects in above- and below-ground processes in a tropical wet forest. Journal of Ecology, 86:597 609.

Lewis, S. L. \& E. V. J. Tanner. 2000. Effects of above- and below-ground competition on growth and survival of rain forest tree seedlings. Ecology, 81: $2525 \sim 2538$.

Ostertag, R.1998. Below-ground effects of canopy gaps in a tropical wet forest. Ecology, 79:1294 1304 .

Strutt, L. T. \& P. A. Keddy. 1996. Above- and below-ground competition intensity in two contrasting wetland plant communities. Ecology, 77: 259 270 .

Vitousek, P. M. \& J. S. Denslow. 1986. Nitrogen and phosphorus availability in treefall gaps of a lowland tropical rainforest. Journal of Ecology, 74: 1167 1178.

Wilson, S. D. \& D. Tilman. 1993. Plant competition and resource availability in response to disturbance and fertilization. Ecology, 74:599 611. 